\title{
Desarrollismo y Humanismo con visión socio-crítica: tendencias pedagógicas en pugna en la Educación Física Argentina del nivel inicial
}

Developmentalism and Humanism with a socio-critical vision: pedagogical tendencies in conflict in the Argentine Physical Education of the initial level

Daniela Mansi

Universidad Nacional de Luján - Universidad de Flores, Argentina

danielamansi@hotmail.com.ar

\begin{abstract}
RESUMEN:
El corriente artículo, encuentra sus bases en la Tesis de Maestría en Actividad Física y Deporte de la Universidad de Flores titulada Construcción Histórica de la Educación Física Argentina del Nivel Inicial.

La pesquisa, se ha sustentado en los supuestos metodológicos de la investigación cualitativa enmarcada en un enfoque interpretativo. El método ha de ser finalístico-intencional, registrando y produciendo análisis a los hechos históricos documentados obtenidos de nuestras fuentes de datos, siendo libros, publicaciones de artículo en la editorial Stadium e investigaciones competentes a la didáctica de la Educación Física del nivel inicial en Argentina.

Proponemos como objetivo de nuestro escrito, analizar y caracterizar dos de las perspectivas ideológicas que nuestra investigación ha arrojado como parte de sus resultados: la visión desarrollista y la visión humanista con sentido socio-crítico de la Educación Física del nivel inicial en Argentina. En relación a ello, generaremos un entramado teórico con las teorías críticas educativas, intentando caracterizar aún más los discursos ideológicos a desarrollar.
\end{abstract}

Palabras claVe: Educación Física, Nivel Inicial, Perspectivas Ideológicas, Hegemonía.

\begin{abstract}
:
This article finds its bases in the Master Thesis in Physical Activity and Sport of the University of Flores entitled Historical Construction of the Argentine Physical Education of the Initial Level. The research has been based on the methodological assumptions of qualitative research, framed in an interpretative approach. The method is finalist-intentional, recording and producing analyzes of the documented historical facts obtained from our data sources, such as books, articles published in the Stadium editorial and competent research in the didactics of Physical Education of the initial level in Argentina. Our aim is to analyze and characterize two of the ideological perspectives that our research has thrown as part of its results: the developmental vision and the humanistic vision with a socio-critical sense of Physical Education at the initial level in Argentina. In relation to this, we will generate a theoretical framework with critical educational theories, trying to further characterize the ideological discourses to be developed.
\end{abstract}

KEYWORDS: Physical Education, Initial Level, Ideological Perspectives, Hegemony.

\section{INTRODUCCIÓN}

El presente escrito, es consecuente a la tesis de Maestría en Actividad Física y Deporte de la Universidad de Flores, la cual ha poseído como tema la Construcción Histórica de la Educación Física en el Nivel Inicial en Argentina. Desde dicha pesquisa, hemos ido analizando las perspectivas ideológicas que han ido conformando la historia de la asignatura, para lograr visibilizar aquellas vertientes dominantes, desarrollando reflexión y construcción de un nuevo discurso sobre la Educación Física en el nivel inicial.

El proceso de investigación, ha arrojado como resultados que la Educación Física Argentina del Nivel Inicial ha sido constituida por las siguientes visiones ideológicas: higienista, deportivista, psicomotricista, desarrollista y humanista con sentido socio-crítico. 
En función de los resultados de la investigación, nos proponemos como objetivo del siguiente escrito analizar en base a las teorías críticas dos perspectivas ideológicas que han inundado a las propuestas corporales en la Educación Física del nivel inicial, siendo ellas: la visión desarrollista y la visión humanista con mirada socio - crítica.

En las siguientes líneas, el/la lector/a podrá encontrarse, con un cierto marco teórico, que le permitirá reconocer cuáles son las bases ideológicas de la perspectiva desarrollista y humanista con sentido socio crítico de la Educación Física en el Nivel Inicial, en articulación a la exposición de ideas provenientes de las teorías críticas.

Generando la matriz conceptual, desarrollaremos brevemente las cualidades del campo de la Educación Física Escolar, específicamente en el nivel inicial. Diferentes discursos que fueron legitimando el área de la Educación Física (EF), han empleado estrategias con el fin de alcanzar la hegemonía del campo. Tomando a Broccoli (1972) nos aclara el concepto gramsciano sobre hegemonía, entendiendo a ésta misma como una relación dialéctica al interior del grupo, categorizando al hombre como el elemento indispensable en todo proceso hegemónico, motivo por el las personas son esencialmente políticos.

Como hemos nombrado anteriormente, en el transcurso de la EF escolar, varios agentes han querido colonizar el campo imponiendo su ideología ${ }^{1}$, léase entre ellos: el discurso proveniente de las ciencias médicas, visión que Bracht (1996) categorizó como higienismo, el discurso de la Institución Militar - surgido a fines del siglo XIX, el discurso deportivo (el cual ha ganado y sostenido la hegemonía del campo) y vertientes provenientes de las ciencias de la Educación, Filosofía y Sociología que han dado un vuelco a la educación.

Todas estas perspectivas ideológicas surgidas en contextos históricos particulares se han encontrado en disputa. En este sentido, Bourdieu (1990) nos aporta que en cualquier campo encontraremos distintas luchas, en los cuales el recién llegado trata de defender su monopolio excluyendo a todo tipo de competencia que haga poner en juego su ortodoxia, es decir, aquellos/as encargados/as del sostenimiento del conservadurismo, opuestos a quienes intentan transformar la ideología del campo.

Remitiéndonos al concepto bourdiano de campo, éste mismo encuentra en su meollo algo que se pone en juego. Dentro del campo de la Educación Física, aquello que se posiciona en el núcleo y genera discusiones y disputas entre sus agentes constitutivos, son los discursos de legitimación dentro de la escuela, y la lucha por introducir o mantener ciertas prácticas corporales.

Nosotros nos detendremos, en las dos visiones nombradas anteriormente, que ha tenido la Educación Física en el nivel inicial, comprendiendo al posicionamiento ideológico a la cual responde cada una de ellas.

\section{Diseño Metodológico}

La investigación ha sido abordada desde un enfoque cualitativo. Siguiendo a Denzin y Lincoln (2011) el término cualitativo implica un énfasis en las cualidades de los entes a investigar, en los procesos y sus significados. Siendo el diseño de tipo exploratorio - descriptivo (Samaja, 1994), enmarcada en un esquema interpretativo, en el cual el objeto a estudiar se lo aborda como un mensaje a descifrar (Ynoub, 2014).

Para nuestra pesquisa, la muestra ha sido finalística - intencional (Ynoub, 2014), nuestras fuentes de datos han de ser libros y artículos de la revista Stadium que han de tener en su cuerpo teórico y propuestas competencias con la Educación Física del Nivel Inicial en Argentina, en relación a nuestro artículo, aquellos hechos históricos documentados que han conformado la perspectiva desarrollista y humanista con sentido socio-crítico de la asignatura. 


\section{Resultados y Discusiones}

\subsection{Perspectiva desarrollista en la Educación Física del Nivel Inicial.}

Recorriendo las huellas de la Educación Física Argentina, para construir el presente escrito, nos detendremos en dos perspectivas que se ha tenido - y tiene- sobre la Educación Física en el nivel inicial, cargadas de ideología que han pisado fuertemente la Educación Física Infantil: la visión desarrollista y la visión humanista con sentido socio - critico.

El recorte realizado corresponde a las dos perspectivas ideológicas, surgidas en los últimos años de la EF en el nivel inicial, que luchan por su espacio en el campo. Desde el tratamiento bourdiano, para que un campo se desenvuelva y funcione, se encuentra la posibilidad que haya algo en juego, un núcleo de movilizaciones que disponga a los/as agentes conformadores/as del campo a jugar, dotado de los hábitus que permitan el conocimiento de las leyes que son inmanentes al juego. Decimos con esto, que el campo de la Educación Física en el nivel inicial, se encuentra conformado por ciertos/as mandatarios/as que se encuentran en pugna, quienes conocen las distintas estrategias de lucha, y empapan a las prácticas de discursos acomodaticios queriendo construir hegemonía.

Para generar claridad a las ideas dichas en líneas anteriores, tomaremos el concepto de ideología, desde Campione (2007), quien, analizando a Gramsci, es vista como la "piel” de la organización social.

Gracias a su constructo conceptual, Gramsci (1981) nos diferencia dos tipos de ideologías. La ideología históricamente orgánica, y la ideología arbitraria. La primera en cuestión tiene una capacidad de organizar grandes masas humanas, conformando el terreno donde los hombres y las mujeres se mueven adquiriendo conciencia. Y las ideologías arbitrarias, crean movimientos individuales.

Dentro del campo de la Educación Física del nivel inicial, tomaremos el concepto gramsciano de ideología históricamente orgánica, ya que sostenemos que las distintas perspectivas han ido generando movimientos crecientes de agentes que conforman el área.

Como primera perspectiva ideológica que ha colonizado el campo de la Educación Física de la primera infancia, tomaremos al desarrollismo. Visión categorizada por Falkenbach, Drexsler y Werle (2006), autoras brasileñas que han llevado a cabo su investigación pedagógica en Educación Física Infantil en cuanto a las concepciones que tienen sobre la misma los/as docentes del área.

Dicha perspectiva que atraviesa las propuestas pedagógicas desarrolladas en el patio escolar, se desprende de la vertiente psicomotricista que ha de surgir en la Argentina en la década del 70, teniendo un alto impacto en la educación del país, específicamente en el nivel inicial y el primer ciclo de la escuela primaria.

Para hablar de la visión desarrollista, creemos propicio en primera instancia, generar algunas aclaraciones conceptuales sobre la psicomotricidad.

Esta trama discursiva, proveniente de la rama psicomotricista, con fundamentación teórica devenida de las ciencias médicas y psicológicas, ha sido pensada como la reeducación psicomotriz de aquellos/as niños/ as que demostraban tener ciertas anomalías motrices. En función de ello, mediante el tratamiento en base a ejercitaciones específicas de las psicomotricidad esos/as niños/as se reeducarían motrizmente, desarrollando aquella faltante que médicos/as de la época creían de necesidad en la niñez.

Quien ha tenido una gran repercusión en nuestra área fue, Le Boulch, médico y profesor de Educación Física, creador del método psikocinético en 1966, el cual "utiliza el movimiento humano para educar" (Le Boulch, 1978, p.11).

Docentes del área de la Educación Física, se apropiaron de contenidos de las psicomotricidad para legitimar su labor en el nivel inicial, conformando de esta manera la perspectiva ideológica nombrada en líneas anteriores: Desarrollista. 
El desarrollismo, es el primer intento de generar una visión autónoma ${ }^{2}$ que atraviesa el patio escolar, que si bien se apropia del discurso psicomotricista, fue pensada desde el meollo de la Educación Física del nivel inicial, y desarrollarado por el corpus de personas que han constituido a la EF del primer nivel educativo.

Como foco central, el desarrollismo tiene entre sus propuestas pedagógicas: el desarrollo de las habilidades motoras básicas, el aumento del acervo motor, y la formación física básica (Giraldes y Porstein, 1976; González y Gómez, 1978; Marrazo y Marrazo, 1970).

Esta forma hegemónica, con patrones dominantes otorgadas al juego, utilizándolo como estrategia metodológica (Rivero, 2011), los "juegos" de salto, de desplazamiento, de puntería, de equilibrio, se convirtieron en la columna vertebral de aquellos libros que circulaban en las cátedras de Educación Física de la educación inicial de los Institutos de Formación Docente. Es así, como "las clases de Educación Física en la infancia se convirtieron de esta manera en un catálogo de ejercitaciones de organización del esquema corporal, el espacio, el tiempo, la lateralidad, etc." (Villa, 2015, p.382).

Desde la corriente desarrollista, se le han otorgado a la Educación Física en el nivel inicial la función de desarrollar las habilidades motrices, pensada además, como estructura favorecedora del deporte escolar. Con respecto al deporte, Gramsci (2009) nos aporta que dentro del él se refleja aquella estructura económico - política de los Estados, siendo la práctica deportiva, una actividad reproductiva de una sociedad individualista. Podemos colocarnos con ojos críticos hacia la nombrada práctica corporal, entendiendo a la misma como un componente que alimenta y reproduce aquellos valores hegemónicos de una sociedad capitalista; el consumismo, la competencia, la jerarquización por rendimiento, la búsqueda del resultado, la individualidad, la mercantilización, cosificación, entre otros.

Los discursos acomodaticios generados por intelectuales defensores/as del desarrollismo, a las teorías educativas críticas del momento, generaron una confusión en el campo de la Educación Física. Tomando a Gómez (2017) quien analiza y desarrolla las ideas gramscianas, específicamente en educación, nos da luz a las categorizaciones sobre los intelectuales que el comunista italiano ha de realizar.

Reforzando la idea que todos y todas somos intelectuales, cumpliendo funciones sociales específicas, una de las categorizaciones propuesta por Giroux (1990) es aclarada como los intelectuales acomodaticios. Gómez (2017) nos revela, lo que compete al sistema educativo, que este tipo de intelectuales se remiten a aquellos/as docentes que de manera acrítica - sin conciencia - de la función social que cumplen desde su labor, tienden a reforzar, reproducir y fomentar los intereses e ideología de las clases dominantes. Estableciendo mediaciones con el campo de la Educación Física podríamos sugerir que estos/as intelectuales, escudándose bajo la objetividad proveniente de las ciencias médicas, tienden a promulgar la corriente positivista decimonónica. Podemos dilucidar y categorizar a aquellos/as docentes sostenedores/as del discurso desarrollista dentro de la EF del nivel inicial, como intelectuales acomodaticios: a) acomodan su discurso teórico a las teorías educativas progresistas, sin ser coherentes con sus propuestas pedagógicas corporales b) argumentan su accionar bajo una mirada psicomotricista, la cual ha nacido desde las corrientes médicas, c) incitan sus prácticas corporales en búsqueda de modelos medibles, eficientes, eficaces y de rendimiento d) fomentan la búsqueda de deportistas bajo la enseñanza de las habilidades motoras básicas. - ¿Básicas para qué? -

La presunción del desarrollo del movimiento en su excelencia, calificado por el/la docente, demuestran un interés en búsqueda de la construcción de un cuerpo infantil empleador.

En función de lo emitido, podemos formular, que la perspectiva ideológica desarrollista, ha conformado la hegemonía del campo, obedeciendo al sentido común. Aquí nos detendremos, en la explicación gramsciana de hegemonía.

Siguiendo a Campione (2007), nos aclara que Gramsci ha extendido aquella noción que Lenin dio sobre el concepto de hegemonía, al cual lo definía como "los mecanismos de la dominación burguesa sobre la clase obrera en la sociedad capitalista” (Campione, 2007, p.74). 
Gramsci (1981) ofrece al concepto de hegemonía una dirección ideológica y cultural, caracterizándolo como una etapa superior, donde los grupos subalternos generan un consenso social en dirección a aquella ideología impuesta por los grupos dominantes.

Retomando la construcción de la hegemonía en el campo de la Educación Física en el Nivel inicial, el desarrollismo ha colocado la mirada en el movimiento y en el cumplimiento del contenido. Sus propuestas disfrazadas de juego, sus consignas en manifestaciones de... ¿A ver quién puede?, ¿A ver quién se anima?, han dilucidado las intenciones de mando, control y adoctrinamiento ideológico, de los/as niños/as, sin aperturas a espacios de despliegue de la creatividad y espontaneidad.

El telón de fondo de las propuestas con perspectivas desarrollistas, se hayan salpicadas por mecanismos de adoctrinamiento que se han de naturalizar pasando desapercibidos.

El rol que ha de tomar el niño y niña, han de ser sumisión a las órdenes y consignas, respeto a la jerarquía (quien ha de ser el/la docente) y hervor por el triunfo incitando a la competencia. "Cuestiones tales como «triunfan los mejores», «que cada palo aguante su vela», «los hombres no lloran», etc., sugieren cómo el sentido común ha incorporado las dimensiones individualistas, insolidarias o machistas de la ideología dominante" (Morgenstern, 1991, p.11).

Las intenciones de desarrollo de juego productivo, que ha de tener dicha perspectiva ideológica, bajo la mirada de un único modelo posible, en búsqueda de la eficiencia, la eficacia y el rendimiento, responden a la ideología dominante de esperarse en un sistema capitalista.

La determinación de propagar infancias productivas en vistas a futuros/as ciudadanos/as pertenecientes al sistema, es el trasfondo de aquellos/as defensores/as, que acríticamente sostienen los alegatos de dicha propuesta.

\subsection{Perspectiva crítica en la Educación Física del nivel inicial. Primeros acercamientos a ideas gramscianas}

"Un programa educativo alternativo no se gesta espontáneamente. Es necesario trabajarlo arduamentepara que llegue y represente a las masas, transformando su conciencia de clase potencial en una conciencia real"

(Morgenstern, 1991, p.10)

Si bien, la perspectiva desarrollista ha de perdurar hasta el día de hoy en los patios escolares, aparece una visión autónoma, que ha dado un vuelco a las propuestas pedagógicas en el nivel inicial: visión humanista con mirada socio - critica.

Con el acento puesto en el sujeto, dicha visión, es consecuencia de la aparición de las teorías críticas educativas. Se propone generar una transformación social por medio de sus propuestas pedagógicas. Creemos que la primera forma de salida de la sumisión es la resistencia, para posteriormente comenzar el proceso de construcción de una nueva hegemonía.

Gómez (2017) nos genera una aclaración con respecto al concepto de hegemonía gramsciano, el cual encierra dos rasgos: por un lado, apoyaba el análisis a los procesos históricos y por otro fundamentaba aquellas intervenciones políticas de los grupos subalternos.

En primera instancia, siguiendo las ideas gramscianas, para lograr construir una nueva hegemonía, debe plantearse una transformación a nivel cultural y educativa. Esta transformación no se logrará de manera individual, sino más bien, en organizaciones de personas que crean y se sostengan en un creciente proyecto de transformación.

En sus Cuadernos de la Cárcel, Gramsci (1981) nos proporciona una analogía entre la guerra de posiciones y guerra de movimientos (estrategias llevadas a cabo en la Primer Guerra Mundial), y aquellas estrategias de emancipación social. 
La guerra de movimientos, donde los/as agentes se conforman como nómades con aspiraciones de desplazamientos rápidos, se contrapone a la idea sostenida por Gramsci sobre la guerra de posiciones y su riqueza en cuanto a las organizaciones sociales asentadas y estáticas. La idea de construir asentamientos fundados genera una estabilidad en la conformación de una contra-hegemonía ${ }^{3}$.

Es decir, para producir transformación se invita a una guerra de posiciones, y pensado desde y para la Educación Física, se incita a la conformación de grupos asentados que reflexionan, sientan y crean posible en una alteración de las prácticas en aristas a una transformación del campo.

Estas ideas gramscianas, en construir un nuevo proyecto por medio de una transformación educativa y cultural, hicieron vibrar a ciertos/as intelectuales del área educativa repensando en una nueva relación pedagógica. Por este motivo, se logró generar un vuelco en la perspectiva ideológica devenida del desarrollismo, para instaurarse en el nivel inicial, una Educación Física crítica - emancipadora de los valores hegemónicos que responden al sistema político, económico y social actual.

La urgencia por contemplar la heterogeneidad de deseos, intereses y gustos de todos/as los/as niños/as, entendidos a estos/as últimos/as como sujetos de derecho, que forman parte cotidianamente de las prácticas en Educación Física, desemboca en la necesidad de garantizar el derecho al juego dentro de la escuela.

Léase el artículo 31 de la Convención de los derechos de la niñez (1989), el cual nos alega que los Estados Partes reconocen el derecho del niño al descanso y el esparcimiento, al juego y a las actividades recreativas propias de su edad y a participar libremente en la vida cultural y en las artes.

A raíz de lo manifestado, los/as intelectuales transformadores (Giroux, 1990) del área, comienzan a pensar en una Educación Física emancipadora, una Educación Física que centre sus propuestas en el sujeto, en sus necesidades, a partir del desarrollo de la autonomía individual y colectiva. Retomando la categorización de Giroux (1990) sobre los intelectuales transformativos, nos esclarece que no sólo son aquellos/as que potencian a sus estudiantes, brindándoles conocimiento y habilidades sociales para actuar en conjunto, sino que enriquecen de sentido crítico educando para la acción transformadora.

La emancipación de aquellas prácticas reproductivas de un sistema capitalista, son desarraigadas de los patios escolares del nivel inicial gracias a esta nueva concepción sobre las infancias (escribimos en plural, entendiendo que no se desenvuelve una sola infancia, sino que cada niño o niña despliega la propia, arraigada a su contexto social, político y económico).

Para ello, se proponen espacios, en donde los/as niños/as tengan la plena libertad de construir situaciones de juegos, tomando al juego y jugar como eje temático, instando el desarrollo del modo lúdico de jugar (Pavía, 2006), es decir, de jugar con valores contra-hegemónicos (Gómez, 2015), o bien, construyendo una nueva hegemonía en lo que respecta a los valores que se desarrollan dentro del juego espontáneo: la cooperación, el compañerismo, la inclusión, el respeto, entre otros.

Esta idea de jugar por jugar (Rivero, 2011), tomando al juego como situación improductiva, fluye contracorriente del juego con intenciones productivas ${ }^{4}$, situación en la cual el juego era una mera actividad motriz, donde el ojo docente se ha encontrado colocado sobre la ejecución de las habilidad motoras. "Los niños pretenden muy natural y justificablemente jugar: encuentran con qué y juegan. Cierto es que no se preocupan por saber si lo que han encontrado, además de útil, también es barato y si el juego merece la pena” (Gramsci, 2009, p.86).

El motor movilizador de las clases de Educación Física en el Nivel Inicial transformadoras, es la opcionalidad, cada niño/a tiene la libertar de elegir a qué jugar, con qué y con quién, propiciando espacios de diálogos democráticos constantes. Se destierran los roles docentes autoritarios y disciplinantes, las imposiciones y obligaciones no tienen lugar en la Educación Física del nivel inicial crítica.

Podríamos pensar, que los ejes centrales de la Educación Física Crítica en el Nivel Inicial son:

- La opcionalidad.

- Preponderancia de la dimensión lúdica (Bracht, 1996) 
- Ambiente favorable a la colaboración (Silva y Bracht, 2012)

- Espacios propicios para el desarrollo del juego y el jugar (Gómez, 2015)

- Exploración, creatividad, imaginación.

- Rol docente abierto, democrático, reflexivo

- Preponderancia del modo lúdico de jugar (Pavía, 2006)

- Autonomía compartida

Con lo expresado anteriormente, la Educación Física Crítica en el nivel inicial, viene de-construyendo aquellas prácticas que se han ido legitimando con anterioridad, para construir nueva teoría y práctica, pensada desde la emancipación a un sistema que se ha filtrado en las paredes de la escuela, para llegar a lo más minúsculo de la tarea docente.

No debe pensarse el surgimiento de una Educación Física Infantil Crítica como consecuencia de una debilidad o error estratégico educativo de las clases dominantes:

Si un sistema educativo se torna ineficiente para contribuir al mantenimiento de la hegemonía de clase, ello no se debe a las características intrínsecas de los aparatos educativos. Más bien habría que pensar que está madurando una situación en la que las clases subalternas se organizan en torno a un proyecto contra-hegemónico, una de cuyas partes esenciales lo constituye un programa educativo de reforma moral e intelectual. (Morgenstern, 1991, p.10)

Es decir, el sistema educativo como instrumento no garantiza completamente la homogeneidad y control social, más bien, se demuestran las intenciones de los grupos subalterno de ejercer un autogobierno, asumiéndose como agentes protagonistas del cambio social.

Este proyecto que viene siendo, sobre transformación de las prácticas pedagógicas en Educación Física del nivel inicial, y su desarrollo contra-hegemónico en la escuela, se consolida en la propuesta gramsciana de conformar intelectuales orgánicos en el núcleo de las clases subalternas (Morgenstern, 1991).

El desarrollo intelectual y físico de aquellos/as integrantes de la clase obrera, es una de las obsesiones que el revolucionario Italiano toma para lograr la transformación social, criticando al espontaneismo en educación.

Con lo que respecta a la Educación Física Crítica en la primera infancia, en líneas anteriores hemos nombrado que la característica principal de ella es la opcionalidad generada a los/as niños/as, por medio de la elección autónoma y espontánea en las clases.

Si nos remitimos al concepto gramsciano, no quisiéramos caer en un espontaneismo que refuerce las líneas autoritarias de la educación sin intervención docente, ya que "librado a la espontaneidad, los hijos de las clases trabajadoras están condenados a vivir y perpetuarse en una cultura subalterna” (Mongenstern, 1991, p.13).

En función de lo emitido, Se proponen en las prácticas de Educación Física del nivel inicial, espacios donde la libertad y la autonomía se hacen visibles, empero la tarea docente se posiciona más fuerte. Sus intervenciones - profesionales -, sus miradas, sus gestos y diálogos democráticos con los/as niños/as, deben de ser el brazo motivador a la emancipación. Al docente de Educación Física podemos verlo/a como guía y acompañante de un proceso pedagógico emancipador y progresista, junto a los/as niños/as protagonistas de su propio proceso de aprendizaje.

\section{Conclusiones}

Hemos realizado con el mayor respeto posible hasta aquí, un entramado conceptual en base a los aportes gramscianos y de distintos/as exégetas que han dado luz a los pensamientos que el comunista italiano ha realizado.

Podemos presumir, que los análisis gramscianos de raíces marxistas, sobre la sociedad ha logrado dar un paso aún mayor a todas las teorías críticas que han devenido durante años. Sus propuestas de estrategia política, no sólo han intentado penetrar en un plano a nivel económico, sino también cavar en lo más 
profundo de aquellas instituciones hegemónicas pertenecientes al Estado (Gómez, 2017) con el fin de ir generando un progresivo desarrollo de transformación social.

Esta apuesta de transformación pedagógica, cultural e ideológica, da cuentas de (re) pensar un proyecto pedagógico contra-hegemónico donde se involucre a los/as niños/as como sujetos plurales, integrales y con emocionalidades singulares.

Esta vocación gramsciana por trasmutar la educación, habría de ser siempre la expresión misma de las clases subalternas, que, bajo la reflexión constante de la eterna dominación de las clases dominantes, han querido organizarse y empoderarse del sistema educativo.

En el área que nos compete en este trabajo, se ha ido analizando aquellas propuestas críticas de promoción de organización popular, pensado ello mismo en la conformación de grupos docentes pogresistas en Educación Física del nivel inicial. Emancipando a los niños y niñas de las reproducciones de prácticas hegemónicas de sostenimiento de la ideología dominante, invitando a re-pensar el proyecto emancipatorio, crítico y progresivo, generando una nueva hegemonía dentro de la Educación Física de la educación inicial en contraposición a la perspectiva desarrollista (la cual ha alimentado a la ideología dominante durante años) que fue colonizando el campo de la Educación Física del nivel inicial.

Han ido brotando en los últimos años algunas experiencias progresistas con aristas a una transformación social. Si bien, desde hace algunas décadas vienen fluyendo movimientos críticos en la Educación Física escolar, aún se encuentra la faltante de forjar intelectuales dentro del área, que se encuentren comprometidos/ as con su labor docente, posicionándose con una mirada crítica de sus prácticas.

Por estos motivos, hemos intentado desnaturalizar aquellos mecanismos de discriminación, segregación y adoctrinamiento que pueden verse en prácticas pedagógicas ancladas en una perspectiva desarrollista.

Se invita abiertamente a todos/as los/as docentes del área, a conformar grupos de estudio, arraigar su voluntad en organizaciones políticas, y criticidad dentro de su labor, para lograr ir generando de esta manera, una nueva Educación Física del nivel inicial, alejada de los valores hegemónicos que responden a las necesidades de un sistema capitalista y (re) pensando un nuevo vínculo pedagógico progresista, llevado a cabo al interior de los grupos subalternos.

\section{Bibliografía}

Asamblea General de las Naciones Unidas. (1989). Convención sobre los Derechos del Niño. Unicef Comité Español. Bourdieu, P. (1990). Sociología y cultura. España: Grijalbo

Bracht, V. (1996) Educación Física y Aprendizaje Social. Córdoba: Vélez Sarsfield.

Campione, D. (2007) Para leer a Gramsci. Buenos Aires: CCC.

Denzin, N. y Lincoln, Y. (2011). Manual de investigación cualitativa. Volúmen I. Barcelona: Gedisa.

Falkenbach, A., Drexsler, G., Werle, V. (2006). Investigando a acao Pedagógica de Educacao Física na Educacao Infantil. Movimento, 12(1), 81-103.

Giraldes, M. y Porstein, A. (1976). El olvidado nivel preescolar. Revista Stadium, 60(10), 45-48

Giroux, H. (1990) Los Profesores como intelectuales. Hacia una pedagogía critica del aprendizaje. Buenos Aires: Paidós.

Gómez, L. (2015) Las intervenciones docentes y la construcción de situaciones lúdicas en la Educación Física Infantil. (Tesis inédita de doctorado). Universitat de Barcelona, España.

Gómez, S. (2017) A 80 años de Antonio Gramsci. Reflexiones políticas y educativas sobre un legado inagotable. Movimento-revista de educação, 4(6), 07-33.

González, L., y Gómez, J. (1978). La educación fisica en la primera infancia. Buenos Aires: Stadium.

Gramsci, A. (1975). La formación de los intelectuales. México: Grijalbo.

Gramsci, A. (1981). Cuadernos de la cárcel. México: Ediciones Era.

Gramsci, A. (2009). Bajo la mole. Fragmentos de civilización. Madrid: Ediciones Sequitur. 
Le Boulch, J. (1978). Hacia una ciencia del movimiento bumano. Introducción a la psikokinética. Buenos Aires: PAIDÓS.

Marrazo, T. y B. de Marrazo, M. (1970). Guía de educación física para los jardines de infantes. Batería de planespara los niveles de cuatro y cinco años. Buenos Aires: Ciordia

Morgenstern, S. (1991). Antonio Gramsci: hegemonía y educación. En F. Gómez de Castro, Socialismo y Sistema Educativo. Madrid: UNED.

Pavía, V. (2006) Jugar de un modo lúdico. El juego desde la perspectiva del jugador. Buenos Aires: Siglo XXI

Rivero, I. (2011). El juego en las planificaciones de Educación Física. Intencionalidad educativa y prácticas docentes. Buenos Aires: Noveduc

Samaja, J. (1999). Epistemología y Metodología. Elementos para una teoría de la investigación cientifica. Buenos Aires: Eudeba.

Silva, M. S., y Bracht, (2012). Na pista de práticas e professores innovadores na educacao física escolar. Kinesis, 30(1), 75-88.

Villa, M. E. (2015). Psicomotricidad. Educación Psicomotriz. En C. Carballo (Coord), Diccionario Crítico de la Educación Física Académica. Rastreo y análisis de los debates y tensiones del campo académico de la educación física en Argentina (págs. 380-384). Buenos Aires: prometeo.

Ynoub, R. (2014). Cuestión de método. Aportes para una metodología crítica. Tomo I. México D.F: CENGAGE Learning.

\section{Notas}

1 Desde los aportes de Campione (2007), quien toma y explica las ideas de Gramsci, esclarece el concepto de ideología, la cual hay que concebirla como una lucha históricamente incesante. La ideología gramsciana repara en las aclaraciones en que la misma se desarrolla: teniendo en la cúspide de la pirámide la filosofía, y por debajo de ella aquellos/as actore/ as divulgadores.

2 Bracht (1996) desarrolla dos tipos de visiones que han de conformar el campo de la EF, por un lado, las heterónomas, es decir, aquellas que se encuentra fuera del campo mismo y han sido tomadas por la EF, y por otro, visiones autónomas, pensadas desde y para la Educación Física.

3 Si bien, Gramsci no remite al concepto contra-hegemonía, es utilizada por algunos/as interpretadores/as de sus aportes.

4 Tipo de juego fomentado por un sistema capitalista, buscando la constante productividad. 